\title{
Bedtime-to-Morning Glucose Difference and iGlarLixi in Type 2 Diabetes: Post Hoc Analysis of LixiLan-L
}

\author{
Ariel Zisman - Terry Dex · Michelle Roberts · Aramesh Saremi • \\ Jason Chao • Vanita R. Aroda
}

Received: June 25, 2018 / Published online: September 14, 2018

(C) The Author(s) 2018

\section{ABSTRACT}

Introduction: A difference of $\geq 50-55 \mathrm{mg} / \mathrm{dL}$ between bedtime and morning glucose (BeAM) values in patients with type 2 diabetes (T2D) on basal insulin is an indicator of poor postprandial glucose control. This analysis compared the effect of treatment with a fixed-ratio combination of insulin glargine/lixisenatide (iGlarLixi) vs insulin glargine (iGlar) on BeAM values, and evaluated the impact of BeAM values on glycemic and safety endpoints.

Methods: In this post hoc analysis of 517 participants from the LixiLan-L trial, change in BeAM values and composite efficacy and safety endpoints stratified by BeAM value $<55 \mathrm{mg} / \mathrm{dL}$ or $\geq 55 \mathrm{mg} / \mathrm{dL}$ were evaluated in patients with

Enhanced digital features To view enhanced digital features for this article go to https://doi.org/10.6084/ m9.figshare.7047896.

\section{A. Zisman ( $($ )}

Endocrine Center of Aventura, Miami, FL, USA

e-mail: azisman@myendocrine.com

T. Dex · M. Roberts - A. Saremi

Sanofi US, Inc, Bridgewater, NJ, USA

J. Chao

Xinyi, Inc, Bridgewater, NJ, USA

V. R. Aroda

Brigham and Women's Hospital, Harvard Medical

School, Boston, MA, USA
T2D uncontrolled on basal insulin randomized to iGlarLixi or iGlar over 30 weeks (LixiLan-L).

Results: Greater reductions in BeAM values were seen with iGlarLixi vs iGlar, and a higher proportion of patients reached a BeAM value $<55 \mathrm{mg} / \mathrm{dL}$ in the iGlarLixi arm. A BeAM value $<55 \mathrm{mg} / \mathrm{dL}$ was associated with improved glycemic control, lower risk of hypoglycemia, and a greater proportion of patients achieving glycemic targets without hypoglycemia or weight gain. Greater reductions in BeAM values were seen with iGlarLixi vs iGlar, irrespective of stratification by glycated hemoglobin $A_{1 c}$ or glycemic endpoints.

Conclusions: Greater reductions in bedtimeto-morning glucose differential, or BeAM, were observed with iGlarLixi vs iGlar in patients with T2D uncontrolled on basal insulin, reflecting better overall control of both fasting and prandial glucose and more appropriate matching of therapy to physiologic needs.

Trial Registration: ClinicalTrials.gov Identifier NCT02058160.

Funding: Sanofi US, Inc.

Keywords: Bedtime-to-morning glucose differential (BeAM); Fasting plasma glucose; Glycated hemoglobin $\mathrm{A}_{1 \mathrm{c}}$; iGlarLixi; Postprandial glucose; Postprandial hyperglycemia; Type 2 diabetes 


\section{INTRODUCTION}

Patients with type 2 diabetes (T2D) who are at, or near, target fasting plasma glucose (FPG) with basal insulin, but not yet at target glycated hemoglobin $A_{1 c}$ (A1C) may be experiencing postprandial glucose (PPG) excursions resulting in residual hyperglycemia [1]. Furthermore, suboptimal treatment of prandial hyperglycemia may result in high bedtime glucose levels. In such cases, it is common clinical practice to continue titration of basal insulin to disproportionally target FPG, which can lead to overbasalization or inappropriately elevated doses of basal insulin, resulting in an increased risk of nocturnal hypoglycemia and weight gain without significantly reducing A1C levels. These side effects, as well as the increasing treatment complexity, can contribute to clinical inertia in escalating therapy beyond basal insulin [2, 3]. Furthermore, real-world data indicate that the probability of patients successfully achieving glycemic goals $(\mathrm{A} 1 \mathrm{C}<7.0 \%)$ substantially diminishes after the first 12 months of initiation of basal insulin [4]. Therefore, understanding when to initiate antihyperglycemic treatment targeting postprandial excursions is vital. Current guidelines recommend consideration of prandial therapy once patients are receiving $>0.5 \mathrm{U} / \mathrm{kg} /$ day of basal insulin [5].

The difference between bedtime and morning glucose levels (BeAM value) is a clinically relevant and easily obtained measure that can be used to help primary care physicians to determine when patients need to start prandial therapy [6]. High BeAM values in T2D patients on basal insulin who have achieved target FPG but still have A1C above target are becoming recognized as an indicator of overbasalization; a study that analyzed BeAM values in 1188 patients with T2D on basal insulin therapy reported a positive correlation between high BeAM values and hyperglycemia, in particular postprandial hyperglycemia. In this study, a BeAM value $\geq 50 \mathrm{mg} / \mathrm{dL}$ was found to indicate a need to initiate prandial therapy [6].

The addition of glucagon-like peptide- 1 receptor agonists (GLP-1 RAs) is a recommended treatment option for patients with T2D on basal insulin with A1C above target despite an acceptable FPG value, and is associated with weight loss and lower hypoglycemia risk compared with prandial insulin [5]. iGlarLixi is a once-daily, titratable, fixed-ratio combination of insulin glargine $100 \mathrm{U} / \mathrm{mL}$ (iGlar), which controls FPG, and the GLP-1 RA lixisenatide, which affects glucose control through enhancement of glucose-dependent insulin secretion, reduction in postprandial glucagon, and slowed gastric emptying $[7,8]$.

\section{Objectives}

The aim of this study was to evaluate the effect of iGlarLixi on BeAM values and to investigate the association between BeAM values and glycemic efficacy endpoints, safety endpoints, and composite endpoints, including both efficacy and safety parameters, in patients with T2D after 30 weeks of treatment.

\section{METHODS}

This was a post hoc analysis of data from the phase 3 LixiLan-L trial (NCT02058160), the full methods of which have been published previously [9]. LixiLan-L was an open-label, randomized, 30-week, parallel-group trial that compared iGlarLixi with iGlar in patients with suboptimal glycemic control on a basal insulin and up to two oral antidiabetes drugs. All patients either continued or were switched to iGlar, and were optimized over a 6-week run-in phase [9]. At the end of the run-in phase, patients who had attained A1C values of $7.0-10.0 \%, \quad F P G \leq 140 \mathrm{mg} / \mathrm{dL}$, and a dose of 20-50 U/day were randomized to once-daily iGlarLixi or iGlar for 30 weeks. iGlarLixi doses were determined by the post-run-in dose of iGlar: if the iGlar dose was $<30 \mathrm{U}$, patients began iGlarLixi at $20 \mathrm{U}$ iGlar/10 $\mu \mathrm{g}$ of lixisenatide (utilizing a 2:1 ratio pen ranging from $10 \mathrm{U} / 5 \mu \mathrm{g}$ to $40 \mathrm{U} / 20 \mu \mathrm{g}$ ); or if the iGlar dose was $\geq 30 \mathrm{U}$, patients began at $30 \mathrm{U}$ iGlar/ $10 \mu \mathrm{g}$ lixisenatide (utilizing a 3:1 ratio pen ranging from $30 \mathrm{U} / 10 \mu \mathrm{g}$ to $60 \mathrm{U} / 20 \mu \mathrm{g}$ ). iGlarLixi and iGlar doses were titrated weekly to a target FPG of $80-100 \mathrm{mg} / \mathrm{dL}$. Because the 
highest available dose of iGlar that can be administered using iGlarLixi is $60 \mathrm{U} /$ day, the iGlar dose in both treatment arms was capped at that level.

All procedures were in accordance with the ethical standards of the responsible committee on human experimentation (institutional and national) and with the Helsinki Declaration of 1964, as revised in 2013. Informed consent was obtained from all patients for study inclusion.

\section{Post Hoc Analysis Design}

BeAM values were determined at baseline and week 30 using 7-point self-measured plasma glucose (SMPG) values. Change in BeAM value from baseline to week 30 and proportion of people reaching a BeAM value $<50 \mathrm{mg} / \mathrm{dL}$ were determined in both iGlarLixi and iGlar treatment groups; in addition, end of study (week 30) glycemic endpoints (A1C $<7.0 \%$, FPG $\leq 100 \mathrm{mg} / \mathrm{dL}$, and PPG $\leq 140 \mathrm{mg} / \mathrm{dL}$ ) were evaluated. Composite efficacy and safety endpoints measured for patients with BeAM values $<55 \mathrm{mg} / \mathrm{dL}$ and $\geq 55 \mathrm{mg} / \mathrm{dL}$ at week 30 in each treatment arm were change in A1C from baseline; proportion of patients with $\mathrm{A} 1 \mathrm{C}<7.0 \%$; composite endpoints of $\mathrm{A} 1 \mathrm{C}<7.0 \%$ without weight gain and/or no documented symptomatic hypoglycemia (defined as typical symptoms of hypoglycemia accompanied by plasma glucose concentration $\leq 70 \mathrm{mg} / \mathrm{dL}$ ); and event rates of documented symptomatic hypoglycemia.

\section{Statistical Methods for Post Hoc Analyses}

$P$ values for continuous variables were calculated using one-way analysis of variance (ANOVA), $P$ values for proportions and incidence rates were calculated using the Pearson $\chi^{2}$ test, and $P$ values for hypoglycemic event rates were calculated on the basis of negative binomial regression with log of exposure as the offset variable. The BeAM values as shown in Table 1 have similar means and SD, and have been tested for normal distribution. The negative values in both groups may have contributed to the wide SDs; however, the number of participants with negative values was similar in both treatment groups and did not affect the analysis outcomes.

\section{RESULTS}

As reported previously, there were no significant differences in demographic or baseline characteristics between the patients using iGlarLixi $(n=259)$ and those using iGlar $(n=258)$ in LixiLan-L [9].

\section{Effect of Treatment on BeAM Value}

By week 30, BeAM values showed significantly greater reductions in patients treated with iGlarLixi vs iGlar $(P<0.001)$, and a higher proportion of people reached a BeAM value $<50 \mathrm{mg} / \mathrm{dL}$ in the iGlarLixi arm compared to the iGlar arm (Table 1). In the iGlarLixi arm, BeAM value declined irrespective of stratification by glycemic endpoints, whereas in the iGlar arm, BeAM value increased slightly except for a decline in those with $\mathrm{A} 1 \mathrm{C} \leq 7.0 \%$ and 2 -h PPG $\leq 140 \mathrm{mg} / \mathrm{dL}$ at week 30 (Table 2).

\section{Impact of BeAM Value on Clinical Outcomes}

In both treatment arms, a BeAM value $<55 \mathrm{mg} /$ $\mathrm{dL}$ was associated with a greater proportion of patients achieving glycemic control (A1C $<7.0 \%)$ at week 30 and each of the composite endpoints (Fig. 1), although treatment with iGlarLixi resulted in a significantly higher percentage of patients achieving glycemic targets and composite endpoints overall compared with those treated with iGlar (Fig. 1a). While pre-breakfast SMPG values were similar for both iGlarLixi and iGlar, there was a trend toward consistently lower bedtime measurements for iGlarLixi compared with iGlar $(P=0.054)$, indicating that the improvement in BeAM values in the iGlarLixi arm compared with the iGlar arm might be due to a lowering of bedtime blood glucose rather than an increase in blood glucose levels in the morning in the majority of patients. In addition, patients treated with 
Table 1 BeAM values for iGlar and iGlarLixi groups

\begin{tabular}{lccc}
\hline & iGlarLixi $(\boldsymbol{n}=\mathbf{2 5 9 )}$ & iGlar $(\boldsymbol{n}=\mathbf{2 5 8})$ & $\boldsymbol{P}$ value $^{\mathbf{a}}$ \\
\hline BeAM values $(\mathrm{mg} / \mathrm{dL})$, mean $(\mathrm{SD})$ & & & \\
Baseline & $58.98(51.18)$ & $54.21(48.23)$ & \\
Week 30 & $43.93(46.45)$ & $55.40(47.21)$ & \\
LS mean change (SE) & $-13.52(2.68)$ & $-0.25(2.68)$ & $<0.001$ \\
BeAM $<50 \mathrm{mg} / \mathrm{dL}$ at week 30, $n(\%)$ & $172(66)$ & $133(52)$ & $<0.001$ \\
\hline
\end{tabular}

To convert $\mathrm{mg} / \mathrm{dL}$ to $\mathrm{mmol} / \mathrm{L}$ use the following formula: $\mathrm{mmol} / \mathrm{L}=(\mathrm{mg} / \mathrm{dL}) / 18$

a $P$ values determined from analysis of covariance with treatment arms (iGlarLixi, iGlar) as fixed effects and baseline analysis value as a covariate

iGlarLixi experienced significantly greater reductions in $\mathrm{A} 1 \mathrm{C}$ compared with patients treated with iGlar, regardless of BeAM value, with numerically larger declines in $\mathrm{A} 1 \mathrm{C}$ in patients with a BeAM value $<55 \mathrm{mg} / \mathrm{dL}$ in both treatment arms (Fig. 1b). A BeAM value $<55 \mathrm{mg} / \mathrm{dL}$ vs $\geq 55 \mathrm{mg} / \mathrm{dL}$ was associated with lower rates of documented symptomatic hypoglycemia at week 30 in both treatment arms (Fig. 1c). iGlarLixi was also associated with a lower rate of documented symptomatic hypoglycemia compared with iGlar irrespective of BeAM values (Fig. 1c).

\section{DISCUSSION}

The significantly greater reduction in BeAM values seen with iGlarLixi compared with iGlar in this post hoc analysis of phase 3 data is consistent with the prandial glucose-targeting effect of the lixisenatide component of iGlarLixi [7]. In the iGlar arm, BeAM values increased among patients who did not reach target $\mathrm{A} 1 \mathrm{C} \leq 7.0 \%$ or 2 -h $\mathrm{PPG} \leq 140 \mathrm{mg} / \mathrm{dL}$ at week 30 , suggesting that BeAM values may be predictive of the need to progress therapy by addressing postprandial hyperglycemia in patients with T2D uncontrolled on basal insulin alone. This highlights the need to identify patients who would benefit from targeted prandial therapy, and indicates that BeAM values may be a suitable new clinical indicator that could be used for this purpose.

This analysis also showed that at week 30 , BeAM values $<55 \mathrm{mg} / \mathrm{dL}$ were associated with better glycemic control and safety endpoints compared with BeAM values $\geq 55 \mathrm{mg} / \mathrm{dL}$, supporting the hypothesis that targeting both FPG and PPG may improve clinical outcomes. This hypothesis was confirmed in a post hoc analysis of LixiLan-L demonstrating that achievement of both FPG and PPG targets with iGlarLixi results in better A1C target attainment compared with FPG or PPG attainment alone [10]. Patients with BeAM values $<55 \mathrm{mg} / \mathrm{dL}$ had less hypoglycemia, regardless of treatment. Higher hypoglycemia rates in patients with BeAM values $\geq 55 \mathrm{mg} / \mathrm{dL}$ may result from titrating basal insulin beyond a dose that can deliver any incremental benefit in achieving A1C targets, and results in precipitating nocturnal hypoglycemia and undesirable weight gain.

\section{Study Limitations}

This study is a post hoc analysis and, as such, its findings should be viewed in the context of hypothesis generation and as a supplement to previous analyses of BeAM values from insulin glargine clinical trials.

\section{CONCLUSIONS}

In this post hoc analysis, iGlarLixi better addressed residual hyperglycemia as demonstrated by a reduction in BeAM values compared with iGlar in patients with T2D uncontrolled on basal insulin. This was also associated with a 
Table 2 BeAM values by treatment arm according to week 30 glycemic endpoints

\begin{tabular}{|c|c|c|c|}
\hline BeAM values, mean $(\mathrm{SD}), \mathrm{mg} / \mathrm{dL}$ & iGlarLixi $(n=259)$ & iGlar $(n=258)$ & $\begin{array}{l}P \text { value }^{\mathrm{a}} \\
\text { (iGlarLixi vs iGlar) }\end{array}$ \\
\hline $\mathrm{A} 1 \mathrm{C} \leq 7.0 \%$ & $(n=171)$ & $(n=90)$ & \\
\hline Baseline & $56.07(53.00)$ & $47.97(41.58)$ & \\
\hline Week 30 & $37.79(40.69)$ & $42.76(40.49)$ & \\
\hline LS mean change (SE) & $-18.54(3.25)$ & $-10.69(4.49)$ & 0.157 \\
\hline $\mathrm{A} 1 \mathrm{C}>7.0 \%$ & $(n=86)$ & $(n=166)$ & \\
\hline Baseline & $64.61(47.23)$ & $57.29(51.34)$ & \\
\hline Week 30 & $56.08(54.86)$ & $61.47(48.66)$ & \\
\hline LS mean change (SE) & $-3.29(4.59)$ & $4.70(3.29)$ & 0.158 \\
\hline $\mathrm{FPG} \leq 100 \mathrm{mg} / \mathrm{dL}$ & $(n=78)$ & $(n=81)$ & \\
\hline Baseline & $57.09(46.53)$ & $60.90(47.92)$ & \\
\hline Week 30 & $43.69(41.68)$ & $61.29(44.34)$ & \\
\hline LS mean change (SE) & $-13.05(4.88)$ & $3.15(4.79)$ & 0.018 \\
\hline $\mathrm{FPG}>100 \mathrm{mg} / \mathrm{dL}$ & $(n=179)$ & $(n=173)$ & \\
\hline Baseline & $59.36(53.16)$ & $51.33(48.64)$ & \\
\hline Week 30 & $43.89(48.68)$ & $53.85(47.92)$ & \\
\hline LS mean change (SE) & $-13.69(3.22)$ & $-0.78(3.28)$ & 0.005 \\
\hline 2-h $\mathrm{PPG}^{\mathrm{b}} \leq 140 \mathrm{mg} / \mathrm{dL}$ & $(n=83)$ & $(n=11)$ & \\
\hline Baseline & $64.04(57.96)$ & $65.07(26.76)$ & \\
\hline Week 30 & $45.37(47.35)$ & $58.84(40.87)$ & \\
\hline LS mean change (SE) & $-14.21(4.56)$ & $-1.16(12.50)$ & 0.327 \\
\hline 2-h $\mathrm{PPG}^{\mathrm{b}}>140 \mathrm{mg} / \mathrm{dL}$ & $(n=167)$ & $(n=235)$ & \\
\hline Baseline, mean (SD) & $57.34(47.37)$ & $53.21(48.98)$ & \\
\hline Week 30, mean $(S D)$ & $41.68(43.69)$ & $55.24(46.98)$ & \\
\hline LS mean change (SE) & $-15.26(3.21)$ & $-0.06(2.71)$ & $<0.001$ \\
\hline
\end{tabular}

For conversion of $\mathrm{mg} / \mathrm{dL}$ to $\mathrm{mmol} / \mathrm{L}$ use the following formula: $\mathrm{mmol} / \mathrm{L}=(\mathrm{mg} / \mathrm{dL}) / 18$

AIC glycated hemoglobin $\mathrm{A}_{1 c}$, BeAM bedtime-to-morning glucose differential, iGlar insulin glargine, iGlarLixi a once-daily titratable fixed-ratio combination of insulin glargine $100 \mathrm{U} / \mathrm{mL}$ (iGlar) and lixisenatide, $P P G$ postprandial glucose, $S D$ standard deviation, $S E$ standard error, $L S$ least squares

a $P$ values determined from analysis of covariance with treatment arms (iGlarLixi, iGlar), analysis variable subgroup, and interaction between treatment and subgroup as fixed effects, and baseline analysis value as a covariate

b Measured $2 \mathrm{~h}$ after a standardized liquid breakfast meal

greater proportion of patients achieving glycemic targets without hypoglycemia or weight gain. The BeAM value has potential as a new and practical clinical decision-making tool to help physicians identify those patients who would benefit from prandial glucose-targeted therapy to improve their glycemic control. 


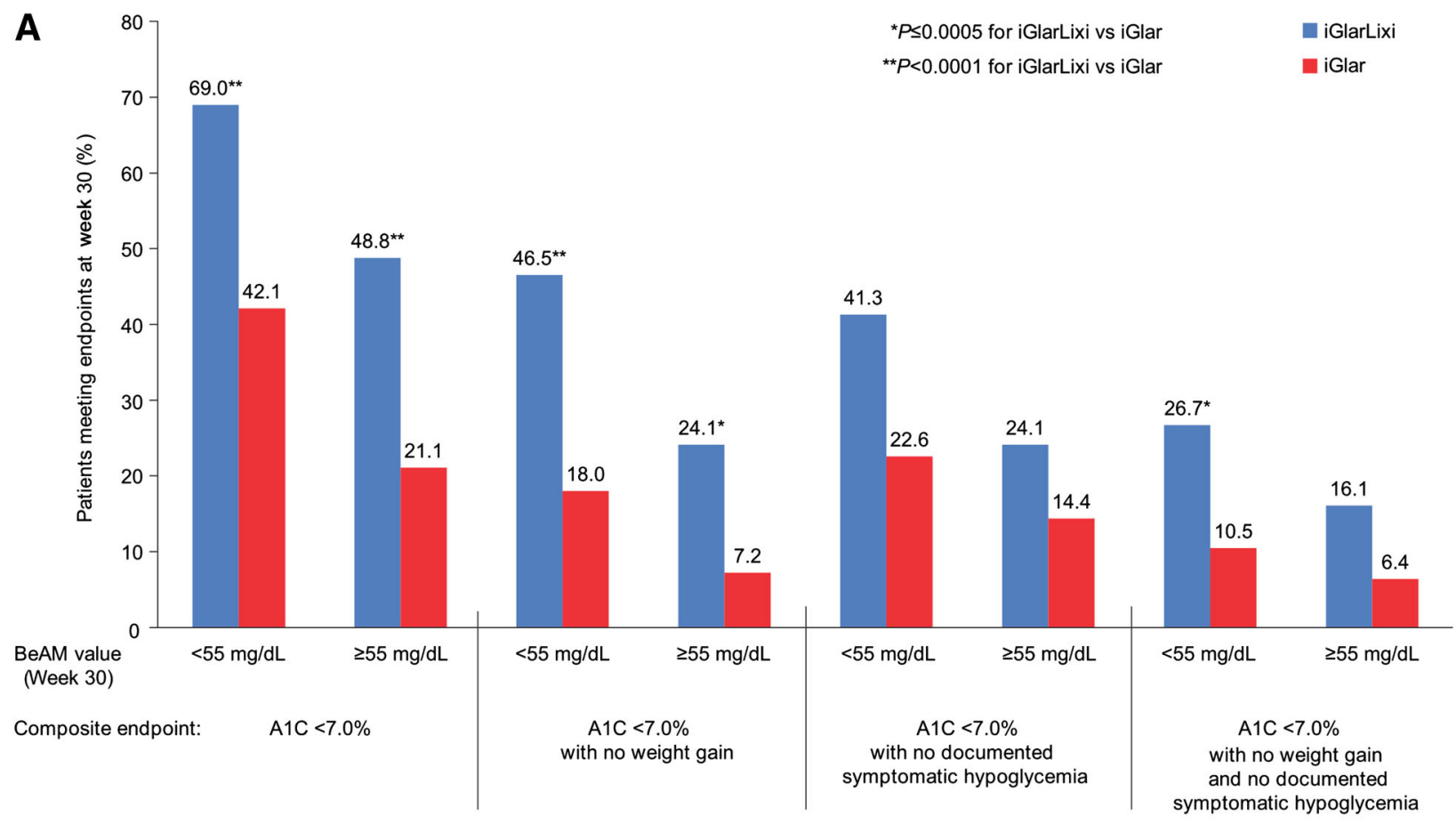

B

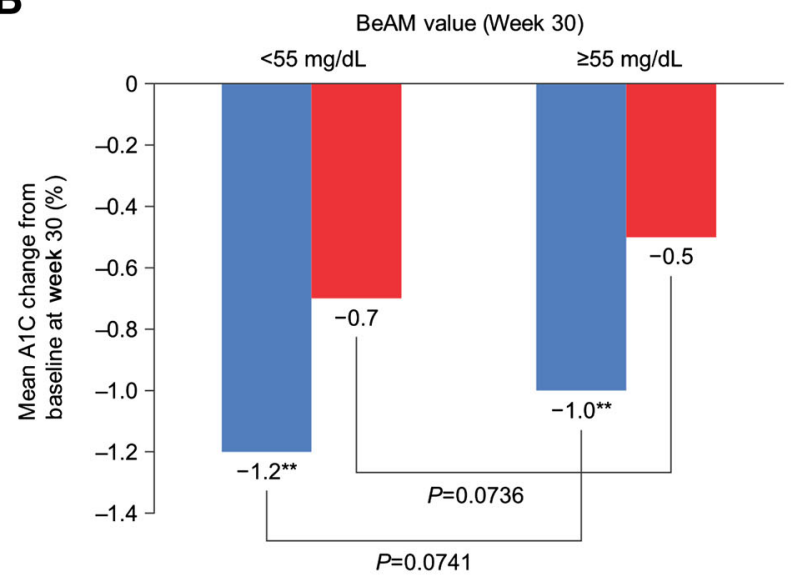

Fig. 1 a Patients achieving target $\mathrm{A} 1 \mathrm{C}$ and composite endpoints at week 30. b A1C change from baseline at week 30. c Documented symptomatic hypoglycemia event rate by week $30 \mathrm{BeAM}$ values $(<55 \mathrm{mg} / \mathrm{dL}$ vs $\geq 55 \mathrm{mg} / \mathrm{dL})$. For conversion of $\mathrm{mg} / \mathrm{dL}$ to $\mathrm{mmol} / \mathrm{L}$ use the following

\section{ACKNOWLEDGEMENTS}

The authors would like to thank all the participants of the LixiLan-L study.
C

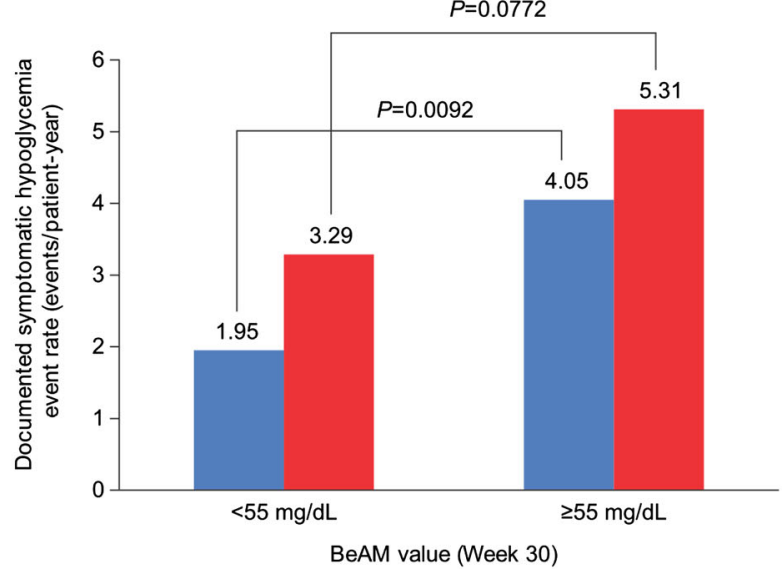

formula: $\mathrm{mmol} / \mathrm{L}=(\mathrm{mg} / \mathrm{dL}) / 18$. A1C glycated hemoglobin $A_{1 c}$, BeAM bedtime-to-morning glucose differential, iGlar insulin glargine $100 \mathrm{U} / \mathrm{mL}$, iGlarLixi a once-daily titratable fixed-ratio combination of iGlar and lixisenatide

Funding. This study and associated article processing charges were funded by Sanofi US, Inc. The study sponsor, Sanofi US, Inc., was involved in all stages of research and 
manuscript preparation; all authors had access to the data, contributed to the manuscript development and take complete responsibility for the integrity of the data and accuracy of the data analysis.

Authorship. All named authors meet the International Committee of Medical Journal Editors (ICMJE) criteria for authorship for this manuscript, take responsibility for the integrity of the work as a whole, and have given final approval to the version to be published.

Medical Writing, Editorial, and Other Assistance. The authors received writing/editorial support from Keisha Peters, MSc, of Excerpta Medica, funded by Sanofi US, Inc. The authors would also like to thank Yan Yan of TechData Service Company, LLC, for assistance with the statistical analyses.

Disclosures. Ariel Zisman has received consultation fees from Sanofi, and is a member of speakers bureaus for AstraZeneca, Novo Nordisk, and Sanofi. Terry Dex is an employee of Sanofi US, Inc. Michelle Roberts is an employee of Sanofi US, Inc. Aramesh Saremi is an employee of Sanofi US, Inc. Jason Chao is an employee of Xinyi, Inc., and under contract with Sanofi. Vanita R. Aroda has had research contracts (clinical trials) within the past 12 months from AstraZeneca/BMS, Calibra, Eisai, Elcelyx, Janssen, Novo Nordisk, Sanofi, and Theracos, and has performed consultant activities within the past 12 months for the American Diabetes Association (ADA), Medscape, Novo Nordisk, Sanofi, and Tufts.

Compliance with Ethics Guidelines. All procedures followed were in accordance with the ethical standards of the responsible committee on human experimentation (institutional and national) and with the Helsinki Declaration of 1964, as revised in 2013. Informed consent was obtained from all individual participants included in the study.

Data Availability. The datasets analyzed during the current study are available from the corresponding author on reasonable request.
Open Access. This article is distributed under the terms of the Creative Commons Attribution-NonCommercial 4.0 International License (http://creativecommons.org/licenses/ by-nc/4.0/), which permits any noncommercial use, distribution, and reproduction in any medium, provided you give appropriate credit to the original author(s) and the source, provide a link to the Creative Commons license, and indicate if changes were made.

\section{REFERENCES}

1. Raccah D, Chou E, Colagiuri S, et al. A global study of the unmet need for glycemic control and predictor factors among patients with type 2 diabetes mellitus who have achieved optimal fasting plasma glucose control on basal insulin. Diabetes Metab Res Rev. 2017;33:e2858.

2. Khunti K, Millar-Jones D. Clinical inertia to insulin initiation and intensification in the UK: a focused literature review. Prim Care Diabetes. 2017;11:3-12.

3. Johnson EL, Frias JP, Trujillo JM. Anticipatory guidance in type 2 diabetes to improve disease management; next steps after basal insulin. Postgrad Med. 2018;130:365-74.

4. Blonde L, Meneghini L, Peng XV, et al. Probability of achieving glycemic control with basal insulin in patients with type 2 diabetes in real-world practice in the USA. Diabetes Ther. 2018;9:1347-58.

5. American Diabetes Association. Standards of medical care in diabetes. Diabetes Care. 2018;41(Suppl. 1):S1-S159.

6. Zisman A, Morales F, Stewart J, Stuhr A, Vlajnic A, Zhou R. BeAM value: an indicator of the need to initiate and intensify prandial therapy in patients with type 2 diabetes mellitus receiving basal insulin. BMJ Open Diabetes Res Care. 2016;4:e000171.

7. Meier JJ, Rosenstock J, Hincelin-Méry A, et al. Contrasting effects of lixisenatide and liraglutide on postprandial glycemic control, gastric emptying, and safety parameters in patients with type 2 diabetes on optimized insulin glargine with or without metformin: a randomized, open-label trial. Diabetes Care. 2015;38:1263-73.

8. Davidson JA. Differential effects of prandial and non-prandial GLP-1 receptor agonists in type 2 diabetes therapy. Postgrad Med. 2015;127:827-41. 
9. Aroda VR, Rosenstock J, Wysham C, et al. Efficacy and safety of LixiLan, a titratable fixed-ratio combination of insulin glargine plus lixisenatide in type 2 diabetes inadequately controlled on basal insulin and metformin: the LixiLan-L randomized trial. Diabetes Care. 2016;39:1972-80.

10. Leiter LA, Chao J, Dex T, Saremi A, Davidson J. A1c target attainment in patients with $\mathrm{T} 2 \mathrm{D}$ receiving
iGlarLixi who reach PPG and FPG targets in the LixiLan-L trial. Abstract presented at the American Diabetes Association 78th Annual Scientific Sessions, 22-26 June, 2018, Orlando, FL, USA. Diabetes. 2018;67(Suppl 1):A290. 